\title{
The sign problem and Abelian lattice duality
}

\author{
Peter Meisinger and Michael Ogilvie ${ }^{* \dagger}$ \\ Washington University, St. Louis \\ E-mail: pnmephysics.wustl.edu,mco@physics . wustl.edu
}

For a large class of Abelian lattice models with sign problems, including the case of non-zero chemical potential, duality maps models with complex actions into dual models with real actions. For extended regions of parameter space, calculable for each model, duality resolves the sign problem for both analytic methods and computer simulations. Explicit duality relations are given for models for spin and gauge models based on $\mathrm{Z}(\mathrm{N})$ and $\mathrm{U}(1)$ symmetry groups. The dual forms are generalizations of the $\mathrm{Z}(\mathrm{N})$ chiral clock model and the lattice Frenkel-Kontorova model, respectively. From these equivalences, rich sets of spatially-modulated phases are found in the strong-coupling region of the original models.

PACS: 11.15.Ha, 11.30.Er, 21.65.0r, 64.60.Cn

31 st International Symposium on Lattice Field Theory LATTICE 2013

July 29 Đ August 3, 2013

Mainz, Germany

* Speaker.

${ }^{\dagger}$ MO gratefully acknowledges the support of the U.S. Dept. of Energy under grant DE-FG02-91ER40628. 
The sign problem is a fundamental issue in Euclidean lattice field theories at non-zero chemical potential, manifesting as complex weights in the path integral [1-3]. We have recently found a prescription that maps a large class of Abelian lattice models with complex weights to dual models with real weights [4]. The dual form of these models can then be studied using familiar analytical and computational methods. The models in this class possess a generalized $\mathscr{P} \mathscr{T}$ symmetry. In recent years, substantial progress has been made in the study of models with this symmetry [57]. The methods developed here are applicable to models with a non-zero chemical potential or a Minkowski-space electric field, which also has a sign problem $[8,9]$. The utility of lattice duality for the sign problem was shown some time ago [10-12], and has recently been systematically studied [13-18] in an intermediate form, particularly in connection with the worm algorithm [19]. The explicit duality relations we derive here based on generalized $\mathscr{P} \mathscr{T}$ symmetry represent a solution to the sign problem for Abelian lattice models over a wide range of parameter space. The dual forms generalize the well-known chiral $Z(N)$ and Frenkel-Kontorova models and typically have a rich phase structure with spatially-modulated phases [20-24]. Such phases are also known to occur in $(1+1)$-dimensional fermionic models [25-30], and would also appear naturally in a quarkyonic phase [31,32]. However, spatially-modulated phases are not special to fermions at finite density, as shown by a continuum model of $(1+1)$-dimensional QCD with heavy particles where the statistics of the particles is immaterial $[33,34,7]$. The appearance of spatially-modulated phases is natural in $\mathscr{P} \mathscr{T}$-symmetric models $[34,7]$.

In the models discussed here, the fundamental fields are elements $z=\exp (i \theta)$ of $Z(N)$ or $U(1)$. The lattice actions are complex, but invariant under the simultaneous application of the operators $\mathscr{C}$ and $\mathscr{T}$, where $\mathscr{C}$ is a linear charge conjugation operator that takes $\theta$ to $-\theta$, and hence $z$ to $z^{*}$, and $\mathscr{T}$ is time reversal implemented as complex conjugation. Thus these models have $\mathscr{C} \mathscr{T}$ symmetry as a generalized $\mathscr{P} \mathscr{T}$ symmetry. In a lattice model, this symmetry ensures that the eigenvalues of the transfer matrix are either real or occur in complex conjugate pairs. The presence of complex eigenvalues gives rise to a rich phase structure not possible with Hermitian transfer matrices $[33,34,7]$.

We begin with duality for $d=2 Z(N)$ models with a chemical potential using the methods of [35] for the Villain, or heat kernel, action. Defining the site-based spin variables as $\exp (2 \pi i m(x) / N)$, with $m(x)$ an integer between 0 and $N$, the partition function is given by

$$
Z\left[J, \mu \delta_{v, 2}\right]=\sum_{m} \sum_{n_{v}} \exp \left[-\frac{J}{2} \sum_{x, v}\left(\frac{2 \pi}{N} \partial_{v} m(x)-i \mu \delta_{v 2}-2 \pi n_{v}(x)\right)^{2}\right]
$$

where $\partial_{v} m(x) \equiv m(x+\hat{v})-m(x)$ and the sum over link variables $n_{v}(x) \in Z$ ensures periodicity. Using the properties of the Villain action, we can write

$$
Z\left[J, \mu \delta_{v, 2}\right]=(2 \pi J)^{-d V / 2} \sum_{m} \sum_{p_{v}} \exp \left[-\frac{1}{2 J} \sum_{x, v} p_{v}^{2}(x)+i \sum_{x, V} p_{v}(x)\left(\frac{2 \pi}{N} \partial_{v} m(x)-i \mu \delta_{v 2}\right)\right]
$$

where $V$ is the number of sites on the lattice such that $d V$ is the number of links. Summation over the $m(x)$ 's give a set of delta function constraints: 


$$
Z\left[J, \mu \delta_{v, 2}\right]=(2 \pi J)^{-d V / 2} \sum_{p_{v}} \exp \left[-\frac{1}{2 J} \sum_{x, V} p_{v}^{2}(x)+\sum_{x, v} p_{2}(x) \mu\right] \prod_{x} \delta_{\partial \cdot p, 0(N)}
$$

where the notation in the Kronecker delta function indicates $\partial \cdot p=0$ modulo $N$. We introduce a dual bond variable $\tilde{p}_{\rho}(X)$ associated with the dual lattice via $p_{v}(x)=\varepsilon_{v \rho} \tilde{p}_{\rho}(X)$ and note that the constraint on $p_{v}$ is solved by $\tilde{p}_{\rho}(X)=\partial_{\rho} \tilde{q}(X)+N \tilde{r}_{v}(X)$. We have

$$
Z\left[J, \mu \delta_{v, 2}\right]=(2 \pi J)^{-d V / 2} \sum_{\tilde{q}, \tilde{r}_{v}} \exp \left[-\frac{1}{2 J} \sum_{x, V}\left(\partial_{\rho} \tilde{q}(X)+N \tilde{r}_{v}(X)\right)^{2}+\mu \sum_{x, V}\left(\partial_{1} \tilde{q}(X)+N \tilde{r}_{1}(X)\right)\right]
$$

which leads to

$$
Z\left[J, \mu \delta_{v, 2}\right]=(2 \pi J)^{-d V / 2} \exp \left[+\frac{V}{2} J \mu^{2}\right] Z\left[\frac{N^{2}}{4 \pi^{2} J},-i \frac{2 \pi J \mu}{N} \delta_{v, 1}\right]
$$

The generalized duality here is

$$
\begin{aligned}
J & \rightarrow \tilde{J}=\frac{N^{2}}{4 \pi^{2} J} \\
\mu \delta_{v, 2} & \rightarrow \tilde{\mu} \delta_{v, 1}=-i \frac{2 \pi J \mu}{N} \delta_{v, 1} .
\end{aligned}
$$

The dual of the original model, which has a complex action, is a chiral $Z(N)$ model with a real action; such models have been extensively studied in two and three dimensions [20-23]. It is convenient to define a parameter $\Delta=J \mu$; the essential characteristics can be understood by considering the range $0 \leq \Delta \leq 1$ [21]. In the limit $\tilde{J} \rightarrow \infty$, i.e., $J \rightarrow 0$, configurations with $\partial_{\rho} \tilde{q}(X)=$ 0 are favored for $\Delta<1 / 2$; this leads to an extension of the ordered phase of the dual model at $\Delta=0$ to non-zero $\Delta$. Beyond $\Delta=1 / 2$, configurations with $\partial_{\rho} \tilde{q}(X) \neq 0$ are favored in the same limit. In two dimensions, this corresponds to phases with a nonzero value of the current coupled to $\mu$. Similar behavior will occur in a broad class of $Z(N)$ models, generalizable to any dimension. In the specific case of a $d=2$ chiral $Z(N)$ model, an incommensurate spatially-modulated phase is found in the $\tilde{J}-\Delta$ plane.

In the interesting case of the $Z(N)$ Villain Higgs model in $d=3$, the partition function has the form

$$
\begin{aligned}
Z\left[J, K, \mu_{v}, G_{v \rho}\right]= & \sum_{m} \sum_{n_{v}} \sum_{p_{v}} \sum_{q_{v \rho}} \exp \left[-\frac{J}{2} \sum_{x, v}\left(\frac{2 \pi}{N} \partial_{v} m(x)-\frac{2 \pi}{N} p_{v}-i \mu_{v}-2 \pi n_{v}(x)\right)^{2}\right] \\
& \times \exp \left[-\frac{K}{2} \sum_{x, v>\rho}\left(\frac{2 \pi}{N}\left(\partial_{v} p_{\rho}-\partial_{\rho} p_{v}\right)-i G_{v \rho}-2 \pi q_{v \rho}\right)^{2}\right]
\end{aligned}
$$

where $\mu_{v}$ is a constant imaginary background vector gauge field that generalizes the chemical potential, and $G_{v \rho}$ is a constant imaginary background field. This model is dual under

$$
\begin{gathered}
J \rightarrow \tilde{J}=\frac{N^{2}}{4 \pi^{2} K} \\
K \rightarrow \tilde{K}=\frac{N^{2}}{4 \pi^{2} J}
\end{gathered}
$$




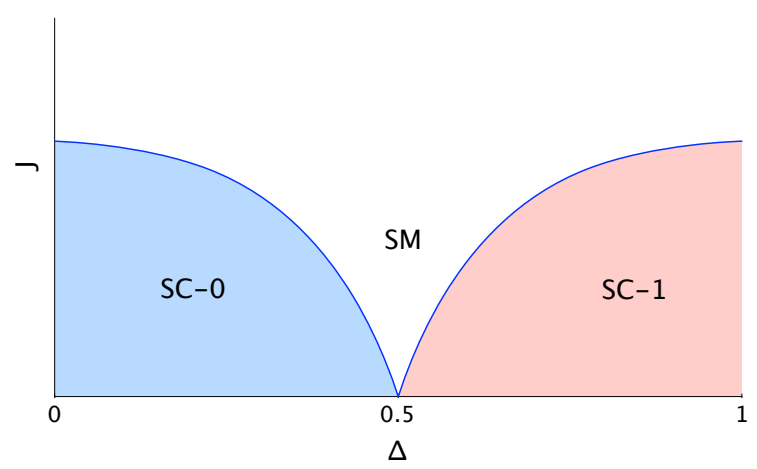

Figure 1: Partial phase diagram for the chiral spin model showing the first two of $N$ homogeneous phases (SC-0 and SC-1) and the region where spatially modulated phases (SM) are found.

$$
\begin{aligned}
\mu_{v} & \rightarrow \tilde{\mu}_{v}=-i \frac{2 \pi K}{N} \varepsilon_{v \rho \sigma} G_{\rho \sigma} \\
G_{v \rho} \rightarrow \tilde{G}_{v \rho} & =-i \frac{2 \pi J}{N} \varepsilon_{v \rho \sigma} \mu_{\sigma}
\end{aligned}
$$

generalizing the well-known self-duality of the $d=3$ Abelian Higgs system. The $d=3 Z(N)$ gauge field is dual to the $d=3$ chiral $Z(N)$ spin model, which has been extensively studied [22,23]. In the strong-coupling limit where $K$ is small and thus $\tilde{J}$ large, the response of the system to an external real (Minkowski-space) electric field reveals an infinite number of commensurate inhomogeneous phases separating the disordered, confining phase of the gauge theory from a phase with a constant induced field.

It is now easy to see how these results generalize to a large class of $Z(N)$ models, classified by their dual forms. Models dual to a $Z(N)$ spin system will have $N$ weak-coupling phases. In the limit $\tilde{J} \rightarrow \infty$, there are transitions between these phases at half-integer values of $\Delta$ where the observable coupled to $\Delta$ jumps. These phases are separated in the $\tilde{J}-\Delta$ plane by spatially-modulated phases. The precise behavior of these spatially-modulated phases depends on both the value of $N$ and the dimensionality $d$. In four dimensions, the dual of the chiral $Z(N)$ model is a less-familiar $Z(N)$ model where the elementary variables are based on plaquettes and the interactions are constructed using cubes. Figure 1 shows a partial phase diagram for the chiral spin model valid for all $N$ and $d \geq 2$. Moving to chiral $Z(N)$ gauge models, we know that in $d=3$, the dual form will be a spin system with a non-zero chemical potential leading to a complex action. In $d=4$, chiral $Z(N)$ gauge models are dual to gauge theories with real Minkowski-space electric fields. It is obvious that there will again be $N$ phases for large $\tilde{K}$, controlled by the parameter $\Gamma=K G$, analogous to $\Delta$. Mean field theory arguments suggest that spatially modulated phases will also be found in this class of models. However, the subtleties associated with gauge degrees of freedom indicate the need for lattice simulations to confirm this behavior. This is especially true for Higgs models, due to charge-screening effects. One case of particular interest is the four-dimensional Higgs model with a chemical potential coupled to the $Z(N)$ scalars. The dual of this model will have elementary link and plaquette variables with plaquette and cube interactions [17].

The duality between $\mathscr{C} \mathscr{T}$-symmetric interactions and chiral interactions is not restricted to the Villain action, but holds for more general actions [4]. There are large regions of parameter space for 
which all the dual weights are positive; such models may be simulated by standard computational methods such as the Metropolis algorithm, and familiar theoretical tools such as mean field theory may be applied. This represents a solution of the sign problem for a large class of Abelian lattice models.

Further insight can be obtained from models based on $U(1)$. Here we apply the duality techniques pioneered by Jose et al. [36]. The partition function of the two-dimensional XY model with an imaginary chemical potential term has the form

$$
Z\left[K, \mu \delta_{v, 2}\right]=\int_{S^{1}}[d \theta] \sum_{n_{v}} \exp \left[-\frac{K}{2} \sum_{x, v}\left(\partial_{v} \theta(x)-i \mu \delta_{v 2}-2 \pi n_{v}(x)\right)^{2}\right] .
$$

Again using the properties of the Villain action, we can apply standard duality techniques [4], finally arriving at

$$
Z=\int_{R}[d \phi(X)] e^{-\sum_{X}\left[\sum_{v}\left(\nabla_{v} \phi(X)\right)^{2} / 2 K+\mu \nabla_{1} \phi(X)\right]} \sum_{\{m(X)\} \in Z} e^{2 \pi i m(X) \phi(X)} .
$$

If we keep only the $m=1$ contributions, we have a lattice sine-Gordon model

$$
Z=\int_{R}[d \phi(X)] \exp \left[-\sum_{X, \mu} \frac{1}{2 K}\left(\nabla_{\mu} \phi(X)\right)^{2}-\sum_{X} \mu \nabla_{1} \phi(X)+\sum_{X} 2 y \cos (2 \pi \phi(X))\right]
$$

with $y=1$. This will be recognized as a two-dimensional lattice version of the Frenkel-Kontorova model, a sine-Gordon model with an additional term proportional to $\mu$. For each fixed value of $X_{2}$, the term $\sum_{X} \nabla_{1} \phi(X)$ counts the number of kinks on that slice: The particles in the original representation manifest as lattice kinks in the dual representation. This generalizes to other lattice models based on $U(1)$, and can also be applied to $Z(N)$ models realized by explicit breaking of $U(1)$ down to $Z(N)$. From a continuum point of view, this model can be further mapped to a massive Thirring model with $\mu$ coupling to the conserved fermion current.

All of the Abelian lattice models in the $\mathscr{C} \mathscr{T}$-symmetric class studied here have real dual representations. These models typically exhibit a rich phase structure in regions of parameter space where the dual weights are positive. The properties of these models can be studied in the dual representation with both computational and analytical tools. Spatially-modulated phases can be detected in simulations using appropriate two-point functions; analytical studies combined with known results from condensed matter physics can provide valuable guidance. Patel has recently suggested that an oscillatory signal might appear in baryon number correlators in heavy ion collisions at RHIC and the LHC $[37,38]$. We believe that the complex phase structure seen in Abelian systems is likely to appear in non-Abelian systems. As an intermediate step, application of duality to an effective Abelian model associated with the reduction of $S U(N)$ to $U(1)^{N-1}$ [39-42] appears possible with the results developed here.

\section{References}

[1] P. de Forcrand, Simulating QCD at finite density, PoS LAT 2009, 010 (2009) [arXiv: 1005 . 0539].

[2] S. Gupta, QCD at finite density, PoS LATTICE 2010, 007 (2010) [arXiv:1101.0109]. 
[3] G. Aarts, Complex Langevin dynamics and other approaches at finite chemical potential, PoS LATTICE 2012, 017 (2012) [arXiv: 1302 . 3028].

[4] P. N. Meisinger and M. C. Ogilvie, The Sign Problem, PT Symmetry and Abelian Lattice Duality, [arXiv:1306.1495].

[5] C. M. Bender, Introduction to PT-Symmetric Quantum Theory, Contemp. Phys. 46, 277 (2005) [quant-ph/0501052].

[6] C. M. Bender, Making sense of non-Hermitian Hamiltonians, Rept. Prog. Phys. 70, 947 (2007) [hep-th/0703096].

[7] P. N. Meisinger and M. C. Ogilvie, PT Symmetry in Classical and Quantum Statistical Mechanics, Phil. Trans. Roy. Soc. Lond. A 371, 20120058 (2013) [arXiv: 1208 . 5077].

[8] E. Shintani, S. Aoki, N. Ishizuka, K. Kanaya, Y. Kikukawa, Y. Kuramashi, M. Okawa and A. Ukawa et al., Neutron electric dipole moment with external electric field method in lattice QCD, Phys. Rev. D 75, 034507 (2007) [hep-lat/0611032].

[9] A. Alexandru and F. X. Lee, The Background field method on the lattice, PoS LATTICE 2008, 145 (2008) [arXiv:0810.2833].

[10] A. Patel, A Flux Tube Model of the Finite Temperature Deconfining Transition in QCD, Nucl. Phys. B 243, 411 (1984).

[11] A. Patel, More on the Flux Tube Model of the Deconfining Transition, Phys. Lett. B 139, 394 (1984).

[12] T. A. DeGrand and C. E. DeTar, Phase Structure of QCD at High Temperature With Massive Quarks and Finite Quark Density: A Z(3) Paradigm, Nucl. Phys. B 225, 590 (1983).

[13] Y. D. Mercado, H. G. Evertz and C. Gattringer, The QCD phase diagram according to the center group, Phys. Rev. Lett. 106, 222001 (2011) [arXiv:1102.3096].

[14] C. Gattringer, Flux representation of an effective Polyakov loop model for QCD thermodynamics, Nucl. Phys. B 850, 242 (2011) [arXiv: 1104 . 2503].

[15] Y. D. Mercado, H. G. Evertz and C. Gattringer, Worm algorithms for the 3-state Potts model with magnetic field and chemical potential, Comput. Phys. Commun. 183, 1920 (2012) [arXiv:1202.4293].

[16] Y. D. Mercado and C. Gattringer, Monte Carlo simulation of the SU(3) spin model with chemical potential in a flux representation, Nucl. Phys. B 862, 737 (2012) [arXiv: 1204 . 6074].

[17] C. Gattringer and A. Schmidt, Gauge and matter fields as surfaces and loops - an exploratory lattice study of the Z(3) Gauge-Higgs model, Phys. Rev. D 86, 094506 (2012) [arXiv: 1208 . 6472 ].

[18] Y. D. Mercado, C. Gattringer and A. Schmidt, Surface worm algorithm for abelian Gauge-Higgs systems on the lattice, Comput. Phys. Commun. 184, 1535 (2013) [arXiv: 1211 . 3436].

[19] N. Prokof'ev and B. Svistunov, Worm Algorithms for Classical Statistical Models, Phys. Rev. Lett. 87, 160601 (2001).

[20] D. Huse, Simple three-state model with infinitely many phases, Phys. Rev. B. 24, 5180-5194 (1981).

[21] S. Ostlund, Incommensurate and commensurate phases in asymmetric clock models, Phys. Rev. B 24, 398 (1981).

[22] J. M. Yeomans and M. E. Fisher, Many commensurate phases in the chiral Potts or asymmetric clock models, J. Phys. C 14, L835-L839 (1981). 
[23] J. M. Yeomans, Low-temperature analysis of the p-state asymmetric clock model for general p, J. Phys. C 15, 7305-7323 (1982).

[24] P. Bak, Commensurate phases, incommensurate phases and the devil's staircase, Rept. Prog. Phys. 45, 587 (1982)

[25] W. Fischler, J. B. Kogut and L. Susskind, Quark Confinement in Unusual Environments, Phys. Rev. D 19, 1188 (1979).

[26] V. Schon and M. Thies, Emergence of Skyrme crystal in Gross-Neveu and 't Hooft models at finite density, Phys. Rev. D 62, 096002 (2000) [hep-th/ 0003195$].$

[27] O. Schnetz, M. Thies and K. Urlichs, Full phase diagram of the massive Gross-Neveu model, Annals Phys. 321, 2604 (2006) [hep-th/0511206].

[28] G. Basar and G. V. Dunne, Self-consistent crystalline condensate in chiral Gross-Neveu and Bogoliubov-de Gennes systems, Phys. Rev. Lett. 100, 200404 (2008) [arXiv: 0803.1501 ].

[29] G. Basar and G. V. Dunne, A Twisted Kink Crystal in the Chiral Gross-Neveu model, Phys. Rev. D 78, 065022 (2008) [arXiv: 0806.2659$]$.

[30] G. Basar, G. V. Dunne and M. Thies, Inhomogeneous Condensates in the Thermodynamics of the Chiral NJL(2) model, Phys. Rev. D 79, 105012 (2009) [arXiv: 0903 . 1868].

[31] T. Kojo, Y. Hidaka, L. McLerran and R. D. Pisarski, Quarkyonic Chiral Spirals,' Nucl. Phys. A 843, 37 (2010) [arXiv:0912.3800].

[32] T. Kojo, Y. Hidaka, K. Fukushima, L. D. McLerran and R. D. Pisarski, Interweaving Chiral Spirals, Nucl. Phys. A 875, 94 (2012) [arXiv: 1107.2124$].$

[33] M. C. Ogilvie and P. N. Meisinger, PT Symmetry and QCD: Finite Temperature and Density, SIGMA 5, 047 (2009) [arXiv:0812.0176].

[34] M. C. Ogilvie, P. N. Meisinger and T. D. Wiser, $\mathscr{P} \mathscr{T}$ Symmetry in Statistical Mechanics and the Sign Problem, International Journal of Theoretical Physics 50, 1042-1051 (2011) [arXiv: 1009.0745$].$

[35] S. Elitzur, R. B. Pearson and J. Shigemitsu, The Phase Structure of Discrete Abelian Spin and Gauge Systems, Phys. Rev. D 19, 3698 (1979).

Phys. Rev. B 3, 3918 (1971).

[36] J. V. Jose, L. P. Kadanoff, S. Kirkpatrick and D. R. Nelson, Renormalization, vortices, and symmetry breaking perturbations on the two-dimensional planar model, Phys. Rev. B 16, 1217 (1977).

[37] A. Patel, Flux Tube Model Signals for Baryon Correlations in Heavy Ion Collisions, Phys. Rev. D 85, 114019 (2012) [arXiv:1111.0177].

[38] A. Patel, Baryon Number Correlations in Heavy Ion Collisions, PoS LATTICE 2012, 096 (2012) [arXiv: 1210.5907$]$.

[39] J. C. Myers and M. C. Ogilvie, New phases of $S U(3)$ and $S U(4)$ at finite temperature, Phys. Rev. D 77, 125030 (2008) [arXiv:0707.1869].

[40] M. Unsal, Abelian duality, confinement, and chiral symmetry breaking in QCD(adj), Phys. Rev. Lett. 100, 032005 (2008) [arXiv: 0708 .1772].

[41] M. Unsal and L. G. Yaffe, Center-stabilized Yang-Mills theory: Confinement and large $N$ volume independence, Phys. Rev. D 78, 065035 (2008) [arXiv: 0803.034 ]].

[42] M. C. Ogilvie, Phases of Gauge Theories, J. Phys. A 45, 483001 (2012) [arXiv: 1211.2843$].$ 